\title{
Predicting NMR Relaxation Rates in Anisotropically Tumbling Proteins through Networks of Coupled Rotators
}

\author{
Gabrielle Nodet, ${ }^{[a]}$ Daniel Abergel, ${ }^{*[a]}$ and Geoffrey Bodenhausen ${ }^{[a, b]}$
}

We show that the prediction of ${ }^{15} \mathrm{~N}$ relaxation rates in proteins can be extended to systems with anisotropic global rotational diffusion by using a network of coupled rotators (NCR), starting from a three-dimensional structure. The relaxation rates predicted by this method are confronted in several examples with experiments performed by other groups. The NCR spectral density func- tions are compared with the results obtained from reduced spectral density mapping. The consequence of the timescales of internal motions on the predicted relaxation rates and the effects of the predicted local anisotropy-present only in the case of anisotropic overall tumbling —on dynamic parameters, are discussed.

\section{Theory}

In a series of recent articles ${ }^{[1-3]}$ the basics of the NCR approach were described in detail, and several applications were presented. In this section we briefly outline its general features and emphasize some aspects that were not discussed previously. The NCR approach provides a simple model for the description of the internal dynamics of a protein provided that 1) the overall and internal motions are statistically decoupled and 2) the internal motions are of limited amplitude.

In this model, internal dynamics are treated in the spaces of the angles formed by internuclear vectors (rotators) that constitute the network. These unitary vectors are parallel to the chemical bonds in the molecule. In order to predict ${ }^{15} \mathrm{~N}-{ }^{1} \mathrm{H}$ dipole-dipole (DD) and ${ }^{15} \mathrm{~N}$ chemical shift anisotropy (CSA) relaxation, one must include the unitary vectors $\mathbf{u}\left(\mathrm{N}_{\mathrm{i}} \mathrm{H}_{\mathrm{i}}\right)$ aligned with the $\mathrm{N}-\mathrm{H}$ bonds. Other types of vectors such as $\mathbf{u}\left(\mathrm{C}_{\mathrm{i}}^{\alpha} \mathrm{H}_{\mathrm{i}}^{\alpha}\right)$, $\mathbf{u}\left(\mathrm{C}_{\mathrm{i}}^{\prime} \mathrm{O}_{\mathrm{i}}\right), \mathbf{u}\left(\mathrm{C}_{\mathrm{i}}^{\prime} \mathrm{C}_{\mathrm{i}}^{\alpha}\right), \mathbf{u}\left(\mathrm{C}_{\mathrm{i}}^{\beta} \mathrm{C}_{\mathrm{i}}^{\gamma}\right), \ldots$ can be incorporated into the network. The time evolution of all vectors can be described by a set of rotational Langevin equations, which can be linearized if the internal motions have small amplitudes and are independent of overall rotational diffusion, thus leading to analytical expressions that are amenable to simple numerical solutions. ${ }^{[1,4]}$

Herein ${ }^{15} \mathrm{~N}$ relaxation was assumed to result from contributions of the ${ }^{15} \mathrm{~N}-{ }^{1} \mathrm{H}$ dipole and ${ }^{15} \mathrm{~N}$ CSA relaxation, and interference effects were not taken into consideration.

\footnotetext{
[a] Dr. G. Nodet, ${ }^{+}$Dr. D. Abergel, Prof. G. Bodenhausen

Département de Chimie, associé au CNRS

Ecole Normale Supérieure

24 Rue Lhomond, 75231 Paris Cedex 05 (France)

Fax: $(+33) 144323397$

E-mail:daniel.abergel@ens.fr

[b] Prof. G. Bodenhausen

Institut des Sciences et Ingénierie Chimiques

Ecole Polytechnique Fédérale de Lausanne

BCH 1015 Lausanne (Switzerland)

$\left.{ }^{+}\right]$Current address:

Institut de Biologie Structurale Jean-Pierre Ebel (CNRS/CEA/UJF)

41, rue Jules Horowitz, 38027, Grenoble (France)
} 
Following Redfield's relaxation theory, ${ }^{[5]}$ longitudinal and transverse ${ }^{15} \mathrm{~N}$ relaxation rates $R_{1 \mathrm{i}}$ and $R_{2 \mathrm{i}}$, and the ${ }^{15} \mathrm{~N}\left\{{ }^{1} \mathrm{H}\right\}$ nuclear Overhauser effect $\eta_{\mathrm{NHi}}$ of each amide group $\mathrm{N}_{\mathrm{i}} \mathrm{H}_{\mathrm{i}}$ are expressed in terms of the auto-correlation spectral density function $J_{\mathrm{ii}}(\omega)$ [Eq. (1)]:

$$
\begin{aligned}
R_{1 \mathrm{i}}= & d^{2}\left[J_{\mathrm{ii}}\left(\omega_{\mathrm{N}}-\omega_{\mathrm{H}}\right)+3 J_{\mathrm{ii}}\left(\omega_{\mathrm{N}}\right)+6 J_{\mathrm{ii}}\left(\omega_{\mathrm{N}}+\omega_{\mathrm{H}}\right)\right]+2 c^{2} J_{\mathrm{ii}}\left(\omega_{\mathrm{N}}\right) \\
R_{2 \mathrm{i}}= & d^{2}\left[2 J_{\mathrm{ii}}(0)+\frac{1}{2} J_{\mathrm{ii}}\left(\omega_{\mathrm{N}}-\omega_{\mathrm{H}}\right)+\frac{3}{2} J_{\mathrm{ii}}\left(\omega_{\mathrm{N}}\right)+3 J_{\mathrm{ii}}\left(\omega_{\mathrm{H}}\right)\right. \\
& \left.+3 J_{\mathrm{ii}}\left(\omega_{\mathrm{N}}+\omega_{\mathrm{H}}\right)\right]+c^{2}\left[\frac{4}{3} J_{\mathrm{ii}}(0)+J_{\mathrm{ii}}\left(\omega_{\mathrm{N}}\right)\right] \\
\eta_{\mathrm{NHi}}= & 1+\frac{\gamma_{\mathrm{H}}}{\gamma_{\mathrm{N}}} \frac{d^{2}\left(6 J_{\mathrm{ii}}\left(\omega_{\mathrm{N}}+\omega_{\mathrm{H}}\right)-J_{\mathrm{ii}}\left(\omega_{\mathrm{N}}-\omega_{\mathrm{H}}\right)\right)}{R_{1}}
\end{aligned}
$$

In these equations, $c_{\mathrm{i}}^{2}=\frac{\left(\gamma_{\mathrm{N}} B_{0} \Delta \sigma_{\mathrm{N}}^{i}\right)^{2}}{15}$ and $d^{2}=\frac{\left(\mu_{0} \hbar \gamma_{\mathrm{N}} \gamma_{\mathrm{H}}\right)^{2}}{10\left(4 \pi\left\langle r_{\mathrm{NH}}^{3}\right\rangle\right)^{2}}, r_{\mathrm{NH}}$ is the average $\mathrm{N}_{\mathrm{i}} \mathrm{H}_{\mathrm{i}}$ distance, $\gamma_{\mathrm{H}}$ and $\gamma_{\mathrm{N}}$ are the ${ }^{1} \mathrm{H}$ and ${ }^{15} \mathrm{~N}$ gyromagnetic ratios, $\mu_{0}$ is the vacuum magnetic susceptibility and $\hbar$ is the reduced Planck constant. The chemical shift anisotropy tensor is assumed to be axially symmetric and identical for all ${ }^{15} \mathrm{~N}$ nuclei, and $\Delta \sigma_{\mathrm{N}}^{\mathrm{i}}=\sigma_{\|}^{\mathrm{i}}-\sigma_{\perp}^{\mathrm{i}}$ denotes the anisotropy. Here $\sigma_{\|}^{\mathrm{i}}$ represents the component of the ${ }^{15} \mathrm{~N}$ chemical shift tensor that is parallel to $\mathbf{u}\left(\mathrm{N}_{\mathrm{i}} \mathrm{H}_{\mathrm{i}}\right)$ and $\sigma_{\perp}^{\mathrm{i}}$ represents its perpendicular components. A detailed analysis of the CSA tensors in ubiquitin has shown that variations for different amide ${ }^{15} \mathrm{~N}$ nuclei are not very large. ${ }^{[6]}$ The spectral density $J_{\mathrm{ii}}$ is the Fourier transform of the auto-correlation function $C_{\text {ii }}(t)$, and is the same for amide ${ }^{15} \mathrm{~N}-{ }^{1} \mathrm{H}$ dipolar and ${ }^{15} \mathrm{~N}$ CSA relaxation. If overall tumbling of the protein is isotropic, the correlation function $C_{\mathrm{ii}}(t)$ can be factorized [Eq. (2)]:

$$
C_{\mathrm{ii}}(t)=C_{0}(t) C_{\mathrm{ii}}^{\mathrm{I}}(t)=\exp \left(-\frac{t}{\tau_{\mathrm{o}}}\right) C_{\mathrm{ii}}^{\mathrm{I}}(t)
$$

where $\tau_{0}$ is the characteristic time of overall rotational diffusion of the protein. Herein, we shall discuss the consequences of anisotropic overall tumbling, where the factorization of Equation (2) is no longer possible. However, in the special case of isotropic overall diffusion, and for small amplitude motions, the internal correlation function $C_{\mathrm{ii}}^{\mathrm{I}}(t)$ is [Eq. (3)]:

$C_{\mathrm{ii}}^{\mathrm{l}}(t)=1-3\left\langle u_{\mathrm{ix}}^{2}+u_{\mathrm{iy}}^{2}\right\rangle^{\mathrm{eq}}+3\left\langle u_{\mathrm{ix}}(0) u_{\mathrm{ix}}(t)+u_{\mathrm{iy}}(0) u_{\mathrm{iy}}(t)\right\rangle$

where $u_{\mathrm{ix}}$ and $u_{\mathrm{iy}}$ are Cartesian components perpendicular to the average (equilibrium) vector $\left\langle\mathbf{u}\left(N_{\mathrm{i}} \mathrm{H}_{\mathrm{i}}\right)\right\rangle .^{\left[{ }^{1]}\right.}$ The order parameter relative to the motions of DD and CSA interactions of the $N_{\mathrm{i}} H_{\mathrm{i}}$ spin pair is $S_{\mathrm{ii}}^{2}=1-3\left\langle u_{\mathrm{ix}}^{2}+u_{\mathrm{iy}}^{2}\right\rangle^{\text {eq }}$. For small-amplitude motions, the Langevin equations describing the dynamics of the network can be linearized, which permits one to derive approximate expressions of the averages $\left\langle u_{\mathrm{ix}}^{2}\right\rangle$ and $\left\langle u_{\mathrm{iy}}^{2}\right\rangle$ and of the correlation functions $\left\langle u_{\mathrm{ix}}(0) u_{\mathrm{ix}}(t)\right\rangle$ and $\left\langle u_{\mathrm{iy}}(0) u_{\mathrm{iy}}(t)\right\rangle$.

The overall NCR potential $U$ results from the sum of pair potentials $U=\sum_{i<j} U_{i j}$ given by Equation (4):

$U_{\mathrm{ij}}=-\frac{1}{2} \kappa_{0} k T \rho_{\mathrm{i}} \rho_{\mathrm{j}}\left[u_{\mathrm{ix}}, u_{\mathrm{iy}}, u_{\mathrm{jx}}, u_{\mathrm{jy}}\right] \mathbf{H}\left[u_{\mathrm{ix}}, u_{\mathrm{iy}}, u_{\mathrm{jx}}, u_{\mathrm{jy}}\right]^{\top}$ where $k$ is the Boltzmann constant, $T$ is the temperature, $\kappa_{0}$ is a common scaling factor that defines the stiffness of the potential, and $\rho_{\mathrm{i}}$ and $\rho_{\mathrm{i}}$ are the local densities in the vicinity of the "reference atoms" of the coupled vectors $\mathbf{u}_{i}$ and $\mathbf{u}_{j}{ }^{\left[{ }^{[1]}\right.}$ The matrix elements of $\mathbf{H}$ in the pair potential are given by the $3 D$ structure of the protein. The NCR gives the correlation functions of the components $u_{\mathrm{ix}}$ and $u_{\mathrm{iy}}$ of the $N$ vectors $\mathbf{u}_{\mathrm{i}}$ comprised in the network, $i=1, \ldots, N$, by the $2 N$ equations [Eq. (5)]:

$\mathbf{V}_{\mathrm{j} \beta}(t)=e^{\mathrm{At}} \mathbf{V}_{\mathrm{j} \beta}(0)$

where the various $\mathbf{V}_{\mathrm{j} \beta}(t)$, with $\beta=x, y$, denote vectors that contribute to the correlation functions, defined in Equation (6):

\begin{tabular}{|c|c|c|c|c|c|c|}
\hline$\left[\left(D_{1} a_{1}^{x x}-2 D_{1}\right)\right.$ & $D_{1} a_{1}^{x y}$ & $D_{1} C_{12}^{2 x}$ & $D_{1} c_{12}^{x y}$ & & $D_{1} C_{1 N}^{x x}$ & $D_{1} c_{1 N}^{x y}$ \\
\hline$D_{1} a_{1}^{x y}$ & $\left(D_{1} a_{1}^{y y}-2 D_{1}\right)$ & $D_{1} C_{12}^{x y}$ & $D_{1} C_{12}^{y y}$ & $\begin{array}{ll}\ldots & \ldots\end{array}$ & $D_{1} C_{1 N}^{y x}$ & $D_{1} C_{1 N}^{y y}$ \\
\hline$\vdots$ & $\vdots$ & & & 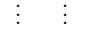 & $\vdots$ & $\vdots$ \\
\hline$D_{\mathrm{N}} c_{\mathrm{N} 1}^{\mathrm{x} x}$ & $D_{N} c_{N 1}^{x y}$ & $\cdots$ & $\cdots$ & $\begin{array}{ll}\cdots & \cdots\end{array}$ & $\left(D_{N} a_{N}^{x x}-2 D_{N}\right)$ & $D_{N} a_{N}^{x y}$ \\
\hline$D_{\mathrm{N}} C_{\mathrm{N} 1}^{x y}$ & $D_{\mathrm{N}} c_{\mathrm{N} 1}^{\mathrm{y}}$ & $\ldots$ & $\cdots$ & $\begin{array}{ll}\cdots & \ldots\end{array}$ & $D_{N} a_{N}^{x y}$ & $\left(D_{N} a_{N}^{d y}-2 D\right.$ \\
\hline
\end{tabular}

$\left\{\begin{array}{l}\mathbf{v}_{\mathrm{jx}}(t)=\left[u_{1 \mathrm{x}}(t) u_{\mathrm{jx}}(0), u_{1 y}(t) u_{\mathrm{jx}}(0), \ldots, u_{\mathrm{Nx}}(t) u_{\mathrm{jx}}(0), u_{\mathrm{Ny}}(t) u_{\mathrm{jx}}(0)\right]^{\top} \\ \mathbf{v}_{\mathrm{jy}}(t)=\left[u_{1 \mathrm{x}}(t) u_{\mathrm{jy}}(0), u_{1 y}(t) u_{\mathrm{jy}}(0), \ldots, u_{\mathrm{Nx}}(t) u_{\mathrm{jy}}(0), u_{\mathrm{Ny}}(t) u_{\mathrm{jy}}(0)\right]^{\top}\end{array}\right.$

with $j=1, \ldots, N$. The matrix $\mathbf{A}$ in Equation (5) is given by Equation $(7)::^{[1]}$

In Equation (7), the coefficients $a_{\mathrm{i}}^{\alpha \beta}, c_{\mathrm{ij}}^{\alpha \beta}$, and so forth, are defined by the equilibrium structure of the molecule through the coupling potential $U$; the diffusion coefficients $D_{\mathrm{i}}$ refer to the individual network vectors $\mathbf{u}_{\mathrm{i}}{ }^{[1]}$ In the simplest version of the NCR model, all vectors are assigned a common value $D_{1}=D_{2}=$ $\ldots=D$. The characteristic diffusion time of the network vectors is defined from the diffusion coefficient $D$ as the time after which the vectors have dephased by $1 \mathrm{rad}$ on average [Eq. (8)]:

$t_{\mathrm{D}}=\frac{1}{6 D}$

Thus $D$ acts as a scaling factor of the timescale of the internal dynamics. The evolution equations can be written in a more compact form by combining the vectors $\mathbf{V}_{\mathrm{j} \beta}(t)$ in a matrix $V$ in Equation (9):

$V(t)=\left[\mathbf{V}_{1 \mathrm{x}}(t), \mathbf{V}_{1 \mathrm{y}}(t), \ldots, \mathbf{V}_{\mathrm{jx}}(t), \mathbf{V}_{\mathrm{jy}}(t), \ldots, \mathbf{V}_{\mathrm{Nx}}(t), \mathbf{V}_{\mathrm{Ny}}(t)\right]$

which obeys the differential equation [Eq. (10)]:

$V(t)=\exp (\mathbf{A} t) V(0)=\mathbf{W}^{\top} \exp \left[\operatorname{diag}\left(\lambda_{1}, \ldots, \lambda_{2 \mathrm{~N}}\right) t\right] \mathbf{W} V(0)$

where $\mathbf{W}$ is the matrix of the eigenvectors of $\mathbf{A}$, and $\left\{\lambda_{i}\right\}$ ( $i=$ $1, \ldots, 2 N)$ are its eigenvalues. The latter are proportional to the common diffusion coefficient $D$ and represent the rotational eigenmodes of the network. In Equation (10), $V(0)$ is the covariance matrix, which completely defines the equilibrium state 
of the network [Eq. (11)].

$$
V(0)=\left[\begin{array}{ccccc}
\left\langle u_{1 x}^{2}\right\rangle^{\text {eq }} & \left\langle u_{1 x} u_{1 y}\right\rangle^{\text {eq }} & \cdots & \left\langle u_{1 x} u_{N x}\right\rangle^{\text {eq }} & \left\langle u_{1 x} u_{N y}\right\rangle^{\text {eq }} \\
\left\langle u_{1 y} u_{1 x}\right\rangle^{\text {eq }} & \left\langle u_{1 y}^{2}\right\rangle^{\text {eq }} & \ldots & \left\langle u_{1 y} u_{N x}\right\rangle^{\text {eq }} & \left\langle u_{1 y} u_{N y}\right\rangle^{\text {eq }} \\
\vdots & \vdots & \vdots & \vdots & \vdots \\
\left\langle u_{\mathrm{Nx}} u_{1 x}\right\rangle^{\text {eq }} & \left\langle u_{\mathrm{Nx}} u_{1 y}\right\rangle^{\text {eq }} & \ldots & \left\langle u_{\mathrm{Nx}}^{2}\right\rangle^{\text {eq }} & \left\langle u_{\mathrm{Nx}} u_{\mathrm{Ny}}\right\rangle^{\text {eq }} \\
\left\langle u_{\mathrm{Ny}} u_{1 x}\right\rangle^{\text {eq }} & \left\langle u_{\mathrm{Ny}} u_{1 y}\right\rangle^{\text {eq }} & \ldots & \left\langle u_{\mathrm{Ny}} u_{\mathrm{Nx}}\right\rangle^{\text {eq }} & \left\langle u_{\mathrm{Ny}}^{2}\right\rangle^{\text {eq }}
\end{array}\right]
$$

Equation (11) is obtained from the NCR model by solving Equation (24) of ref. [1]. Hence, the correlation functions $C_{\mathrm{ii}}(t)$ can readily be calculated from Equations (2), (3), and (10) and subsequently Fourier transformed to yield the spectral density functions $J_{\mathrm{ii}}^{N C R}$ [Eq. (12)]:

$J_{\mathrm{ii}}^{\mathrm{NCR}}(\omega)=\int_{0}^{\infty} C_{\mathrm{ii}}(t) \cos (\omega t) d t$

Herein, the theory outlined above will be generalized to the case of anisotropic overall tumbling, where the correlation functions cannot be factorized as in Equation (2). In this case, the rotational diffusion of the protein as a whole modulates the effects of internal dynamics on NMR relaxation, and the spin relaxation rates depend on the orientation of the $\mathbf{u}\left(\mathrm{N}_{\mathrm{i}} \mathrm{H}_{\mathrm{i}}\right)$ vectors with respect to the principal axes of the protein diffusion tensor. ${ }^{[7,8]}$

For an overall diffusion described by a symmetric top model the correlation function $C_{\mathrm{ii}}(t)$ is given in Equation (13), using the conventions used by Daragan and Mayo: ${ }^{[9]}$

$$
\begin{aligned}
C_{\mathrm{ii}}(t) & =\sum_{\mathrm{r}=0}^{\mathrm{r}=+2} e^{-\mathrm{D}_{\mathrm{r}} \mathrm{t}}\left[\xi_{\mathrm{ir}, 1}\left(1-3\left\langle u_{\mathrm{ix}}^{2}+u_{\mathrm{iy}}^{2}\right\rangle^{\text {eq }}\right)+\xi_{\mathrm{ir}, 2}\left\langle u_{\mathrm{ix}}^{2}-u_{\mathrm{iy}}^{2}\right\rangle^{\text {eq }}\right. \\
& +3 \xi_{\mathrm{ir}, 3}\left\langle u_{\mathrm{ix}}(0) u_{\mathrm{ix}}(t)+u_{\mathrm{iy}}(0) u_{\mathrm{iy}}(t)\right\rangle \\
& \left.+\xi_{\mathrm{ir}, 4}\left\langle u_{\mathrm{ix}}(0) u_{\mathrm{ix}}(t)-u_{\mathrm{iy}}(0) u_{\mathrm{iy}}(t)\right\rangle\right]
\end{aligned}
$$

where the symbol $D_{\mathrm{r}}$ denotes the three eigenvalues of the overall diffusion tensor and the coefficients $\xi_{i, s}$ relate the orientation of the vector $\mathbf{u}\left(\mathrm{N}_{\mathrm{i}} \mathrm{H}_{\mathrm{i}}\right)$ to the diffusion tensor frame. The expressions of $\xi_{\text {ir, }}$ are defined by Daragan and Mayo ${ }^{[9]}$ and are reproduced in Table 1. In Equation (13), one recognizes the term $1-3\left\langle u_{\mathrm{ix}}^{2}+u_{\mathrm{iy}}^{2}\right\rangle^{\text {eq }}$ that corresponds to the order parameter $S_{\mathrm{ii}}^{2}$ for isotropic global motion. The additional contributions of $\left\langle u_{i x}^{2}-u_{i y}^{2}\right\rangle^{\text {eq }}$ arise from the anisotropy of internal motions. Note that these are revealed only when the overall tumbling of the protein is anisotropic. Since $\sum_{\mathrm{r}=0}^{2} \xi_{\mathrm{ir}, 2}=0$, these contributions vanish at $t=0$. They are expected to be non-negligible only at intervals long enough that the contributions from the different terms $e^{-D_{\mathrm{r}} \mathrm{t}}, r=0,1,2$, become significantly different. Thus, for an anisotropically tumbling protein, spin relaxation depends on the protein structure in two different ways in the NCR approach: through the coupling potential, which determines the averages of these vector fluctuations; and through the orientation of the equilibrium vectors with respect to the overall diffusion tensor, as expressed by the coefficients $\xi_{\mathrm{i}, \mathrm{s}}$ in Table 1. However, the effects of internal motions on relaxation are masked by those of global motions at long times, and the contributions of local anisotropy to ${ }^{15} \mathrm{~N}$ relaxation are therefore expected to be very small.

\section{Experimental Section}

The Network: Recent improvements in the computing procedure allowed us to speed up computations and therefore to use up to eight different types of vectors in the calculations: $\mathbf{u}\left(\mathrm{N}_{\mathrm{i}} \mathrm{H}_{\mathrm{i}}\right), \mathbf{u}\left(\mathrm{C}_{\mathrm{i}}^{\alpha} \mathrm{H}_{\mathrm{i}}^{\alpha}\right)$, $\mathbf{u}\left(C_{i}^{\prime} \mathrm{O}_{i}\right), \mathbf{u}\left(\mathrm{C}_{i}^{\prime} \mathrm{C}_{\mathrm{i}}^{\alpha}\right), \mathbf{u}\left(\mathrm{C}_{\mathrm{i}}^{\alpha} \mathrm{C}_{\mathrm{i}}^{\beta}\right), \mathbf{u}\left(\mathrm{C}_{i}^{\beta} \mathrm{C}_{\mathrm{i}}^{\gamma}\right), \mathbf{u}\left(\mathrm{C}_{\mathrm{i}}^{\prime} \mathrm{N}_{\mathrm{i}+1}\right), \mathbf{u}\left(\mathrm{C}_{i}^{\alpha} \mathrm{N}_{\mathrm{i}}\right)$ and $\mathbf{u}\left(\mathrm{Y}_{\mathrm{i}} \mathrm{Z}_{i}\right)$ where $Y_{1}$ and $Z_{i}$ denote atoms located in the side-chains, as shown in Table 2. This improvement does not lead to prohibitive computation times (a few minutes on a PC) and in principle allows for the investigation of side chains. Using a network comprising a large number of vector types made it necessary to adapt some of the parameters in the potential. Thus, a cut-off distance $R_{\mathrm{c}}=5 \AA$ and a scaling factor $\kappa_{0}=2.5$ were used in all calculations in this work. The choice of $t_{\mathrm{D}}$ for the network is discussed below.

Correlation Functions: The overall tumbling correlation time $\tau_{0}$ [or, for anisotropic tumbling, the overall diffusion tensor eigenvalues $\left.D_{\mathrm{r}}(r=1,2,3)\right]$ were determined from the ratios $R_{2} /$ $R_{1}$, using established methods. ${ }^{[10]}$ In the case of isotropic
Table 2. Vector types in sidechains of various amino acid residues.

\begin{tabular}{|ll} 
Residue & Vector $\mathbf{u}\left(Y_{i} Z_{i}\right)$ \\
\hline LEU & $\mathbf{u}\left(C_{i}^{\gamma}-C_{i}^{\delta 1}\right)$ \\
& $\mathbf{u}\left(C_{i}^{\gamma}-C_{i}^{\delta 2}\right)$ \\
MET & $\mathbf{u}\left(S_{i}^{\delta}-C_{i}^{18}\right)$ \\
GLU & $\mathbf{u}\left(C_{i}^{\delta}-O_{i}^{\varepsilon 1}\right)$ \\
GLN & $\mathbf{u}\left(C_{i}^{\delta}-O_{i}^{\varepsilon 1}\right)$ \\
LYS & $\mathbf{u}\left(C_{i}^{\varepsilon}-N_{i}^{\delta}\right)$ \\
ARG & $\mathbf{u}\left(C_{i}^{\zeta}-N_{i}^{\eta 1}\right)$ \\
TYR & $\mathbf{u}\left(C_{i}^{\zeta}-O_{i}^{\eta 1}\right)$ \\
\hline
\end{tabular}
overall tumbling, the internal correlation functions $C_{\mathrm{ii}}^{1}(t)$ were calculated from $\left\langle u_{\mathrm{ix}}(t) u_{\mathrm{ix}}(0)\right\rangle$ and $\left\langle u_{\mathrm{iy}}(t) u_{\mathrm{iy}}(0)\right\rangle$ by numerical evaluation of Equations (3) and (10-11). Due to the presence of overall tumbling, the characteristic diffusion time $t_{\mathrm{D}}$ does not lead to a mere scaling of the relaxation rates. Rather, it is the ratio $t_{\mathrm{D}} / \tau_{0}$ that affects the spin relaxation rates. The time step used in the calculations is small enough to describe correctly the initial part of the correlation function, where variations are mainly due to fast internal dynamics. Calculations using Equation (10) extended to $5 t_{D}$; at which point $C_{\mathrm{ii}}^{\prime}(t)$ was observed to have reached a plateau value numerically, and could therefore safely be assumed stationary, so that for $t \geq 5 t_{\mathrm{D}}, C_{\mathrm{ii}}^{1}(t)$ was replaced by its equilibrium value $S_{\mathrm{ii}}^{2}$. Calculations of the total correlation 
functions $C_{\mathrm{ii}}(t)$ using Equation (2) were then extended to times of up to $5 \tau_{0}$ and Fourier transformed. The resulting spectral density functions $J_{\text {ii }}(\omega)$ at frequencies $\omega=$ $0, \omega_{\mathrm{N}}, \omega_{\mathrm{H}}, \omega_{\mathrm{H}} \pm \omega_{\mathrm{N}}$, which are relevant to Equation (1), were determined by spline interpolation. All calculations were performed using Scilab software. ${ }^{[11]}$

For anisotropic global rotational diffusion, the correlation functions $\left\langle u_{\mathrm{ix}}(t) u_{\mathrm{ix}}(0)\right\rangle$ and $\left\langle u_{\mathrm{iy}}(t) u_{\mathrm{iy}}(0)\right\rangle$ were calculated in a similar way and their values were inserted in Equation (13) to provide the total correlation function $C_{i i}(t)$. Calculations were performed for times $t \leq$ $5 \times \max \left(\frac{1}{6 D_{\mathrm{r}}}\right)$.

Spectral Density Mapping: The spectral density mapping (SDM) analysis of relaxation rates $^{[12,13]}$ consists in extracting the values of the spectral density functions only at frequencies that contribute to the relaxation rates, that is at $J_{\mathrm{ii}}(0), J_{\mathrm{ii}}\left(\omega_{\mathrm{N}}\right), J_{\mathrm{ii}}\left(\omega_{\mathrm{H}}\right), J_{\mathrm{ii}}\left(\omega_{\mathrm{H}} \pm \omega_{\mathrm{N}}\right)$. This approach was adapted to situations where only three independ-

ent measurements are available. In this so-called reduced spectral density mapping ( $\mathrm{rSDM}$ ), the high-frequency contributions to relaxation rates are assumed to be equal: $J_{\mathrm{ii}}\left(\omega_{\mathrm{h}}\right)=J_{\mathrm{ii}}\left(\omega_{\mathrm{H}}\right)=J_{\mathrm{ii}}\left(\omega_{\mathrm{H}} \pm \omega_{\mathrm{N}}\right) \cdot{ }^{[14-}$ 16] The spectral density functions at three frequencies, $J_{\mathrm{ii}}^{\mathrm{SDM}}(0)$, $\int_{\mathrm{ii}}^{\mathrm{SDM}}\left(\omega_{\mathrm{N}}\right)$ and $\int_{\mathrm{ii}}^{\mathrm{SDM}}\left(\omega_{\mathrm{h}}\right)$ can be extracted from three ${ }^{15} \mathrm{~N}$ relaxation measurements for each residue $i$ [Eq. (14)]:

$$
\left\{\begin{array}{l}
\int_{\mathrm{ii}}^{\mathrm{SDM}}\left(\omega_{\mathrm{h}}\right)=\frac{R_{\mathrm{i}}}{5 d^{2}} \frac{\gamma_{\mathrm{N}}}{\gamma_{\mathrm{H}}}\left(\eta_{\mathrm{NHi}}-1\right) \\
\int_{\mathrm{ii}}^{\mathrm{SDM}}\left(\omega_{\mathrm{N}}\right)=\frac{R_{\mathrm{i}}}{3 d^{2}+2 c^{2}}\left(1-\frac{7 \gamma_{\mathrm{N}}}{5 \gamma_{\mathrm{N}}}\left(\eta_{\mathrm{NHi}}-1\right)\right) \\
\int_{\mathrm{ii}}^{\mathrm{SDM}}\left(\omega_{\mathrm{N}}\right)=\frac{1}{4\left(3 d^{2}+2 c^{2}\right)}\left[6 R_{2 \mathrm{i}}-R_{1 \mathrm{i}}\left(3+\frac{18 \gamma_{\mathrm{N}}}{5 \gamma_{\mathrm{N}}}\left(\eta_{\mathrm{NHi}}-1\right)\right)\right]
\end{array}\right.
$$

According to Redfield's theory, the right hand side in the first line of Equation (14) should be equal to [Eq. (15)]:

$\frac{R_{1 \mathrm{i}}}{5 d^{2}} \frac{\gamma_{\mathrm{N}}}{\gamma_{\mathrm{H}}}\left(\eta_{\mathrm{NHi}}-1\right)=\frac{6 J_{\mathrm{ii}}\left(\omega_{\mathrm{N}}+\omega_{\mathrm{H}}\right)-J_{\mathrm{iii}}\left(\omega_{\mathrm{N}}-\omega_{\mathrm{H}}\right)}{5}$

[see Eq. (1)]. Thus the high-frequency term $\int_{\mathrm{ii}}^{\mathrm{SDM}}\left(\omega_{\mathrm{h}}\right)$ can be compared quite naturally to the quantity $\int_{\mathrm{ii}}^{N C R}\left(\omega_{\mathrm{h}}\right)$ defined as [Eq. (16)]:

$J_{\mathrm{ii}}^{\mathrm{NCR}}\left(\omega_{\mathrm{h}}\right) \frac{6 J_{\mathrm{ii}}^{\mathrm{NCR}}\left(\omega_{\mathrm{H}}+\omega_{\mathrm{N}}\right)-J_{\mathrm{ii}}^{\mathrm{NCR}}\left(\omega_{\mathrm{H}}-\omega_{\mathrm{N}}\right)}{5}$

which can be calculated through the NCR approach.

Chemical Exchange: The decay of transverse magnetization is determined by the rate $R_{2}^{\text {app }}=R_{2}^{\text {true }}+R_{\text {ex }}$ that is, by a combination of relaxation $R_{2}^{\text {true }}$ and exchange broadening $R_{\text {ex }}$. Transverse relaxation rates $R_{2}$ are sensitive to internal motions that occur on timescales shorter than the overall tumbling time $\tau_{0}$ of the molecule. In contrast, chemical exchange contributions $R_{\text {ex }}$ to the decay of transverse magnetization originate from chemical shift modulations that occur on timescales of $\mu \mathrm{s}-\mathrm{ms}$, reflecting local fluctuations of the electronic environment caused by internal motions. Clearly, exchange contributions cannot be predicted by the NCR model. They must be subtracted from the apparent relaxation rates $R_{2 \mathrm{i}}^{\text {app }}$ of the ${ }^{15} \mathrm{~N}$ nucleus of the $i^{\text {th }}$ residue before comparing $R_{2 \mathrm{i}}^{\text {true }}=R_{2 \mathrm{i}}^{\text {app }}-R_{\text {exi }}$ with the rates $R_{2 \mathrm{i}}^{\mathrm{NCR}}$ predicted from NCR theory.

\section{Results}

\subsection{Predicting Relaxation Rates in Isotropically Tumbling Proteins by NCR-Comparison with Reduced Spectral Density Mapping (rSDM)}

Now we present an extension of previous findings, ${ }^{[3]}$ and illustrate the potential of our approach with several examples. In order to confront our theory with experimental measurements, we predicted the ${ }^{15} \mathrm{~N}$ relaxation rates $R_{1 \mathrm{i}}^{\mathrm{NCR}}, R_{2 \mathrm{i}}^{\mathrm{NCR}}$ and $\eta_{\mathrm{NHi}}^{\mathrm{NCR}}$ for the proteins Lysozyme ${ }^{[17]}$ and Interleukin- $4^{[18]}$ and compared them to the experimental rates. The simple dynamic model of Equation (3) was used and the network comprised eight types of vectors (instead of only four ${ }^{[3]}$ ), based on the NMR structures of the proteins. ${ }^{[19,20]}$ The characteristic time of internal diffusion was set to $t_{\mathrm{D}}=300 \mathrm{ps}$. The $R_{\text {exi }}$ contributions (36 in Interleukin$4,{ }^{[18]} 30$ in Lysozyme ${ }^{[17]}$ ) were subtracted from the apparent rates $R_{2 \mathrm{i}}^{\text {app }}$ and our predictions $R_{2 \mathrm{i}}^{\mathrm{NCR}}$ were compared with the corrected experimental rates $R_{2 \mathrm{i}}^{\text {true }}=R_{2 \mathrm{i}}^{\mathrm{app}}-R_{\text {exi, }}$ as shown in Figure 1. The agreement between our predictions and the experiments was estimated by several complementary criteria: Spearman rank-order and linear correlation coefficients $\rho_{\mathrm{s}}$ and $\rho_{1}{ }^{[21]}$ and root mean-square deviations $\sigma$, as shown in Table 3. In addition, a moving average $\sigma^{\mathrm{m}}$ of the root mean square deviation was calculated over a window of five neighbouring res- 


\begin{tabular}{|c|c|c|c|c|c|c|}
\hline \multirow{2}{*}{$\begin{array}{l}\text { Protein } \\
\text { Coefficient }\end{array}$} & \multicolumn{3}{|c|}{ Lysozyme } & \multicolumn{3}{|c|}{ Interleukin-4 } \\
\hline & $\rho_{\mathrm{s}}$ & $\rho_{\mathrm{l}}$ & $\sigma$ & $\rho_{\mathrm{s}}$ & $\rho_{1}$ & $\sigma$ \\
\hline$R_{2 \mathrm{i}}^{\mathrm{NCR}}$ vs $R_{2 \mathrm{i}}^{\text {true }}$ & 0.61 & 0.65 & 0.57 & 0.69 & 0.81 & 0.69 \\
\hline$R_{1 \mathrm{i}}^{\mathrm{N} C R}$ vs $R_{1 \mathrm{i}}^{\text {exp }}$ & 0.55 & 0.63 & 0.14 & 0.54 & 0.75 & 0.13 \\
\hline$\eta_{\mathrm{NHi}}^{\mathrm{NCR}}$ vs $\eta_{\mathrm{NHH}}^{\exp }$ & 0.12 & 0.03 & 0.16 & 0.75 & 0.77 & 0.17 \\
\hline
\end{tabular}

idues (see Figure 2). It is clear from this figure that large values of $\sigma^{\mathrm{m}}$ are associated with loop regions of the proteins. Interestingly, for both proteins, the rates predicted for each ensemble of structures exhibit large dispersions in these loops, which may be due to the lack of accuracy of the NMR structure in these regions. These results emphasize the crucial role of structure in the NCR approach.

The rSDM analysis based on amide ${ }^{15} \mathrm{~N}$ experimental relaxation rates $R_{2 \mathrm{i}}^{\text {true }}, R_{1 \mathrm{i}}^{\text {exp }}, \eta_{\mathrm{NHi}}^{\text {exp }}$ was compared to the spectral density functions predicted by the NCR approach. Again, the statistical agreement between predicted spectral densities $J_{\mathrm{ii}}^{\mathrm{NCR}}(0)$, $J_{\mathrm{ii}}^{\mathrm{NCR}}\left(\omega_{\mathrm{N}}\right), \quad J_{\mathrm{ii}}^{\mathrm{NCR}}\left(\omega_{\mathrm{h}}\right), \quad$ and obtained from experiments, $\int_{\mathrm{ii}}^{\mathrm{SDM}}(0), J_{\mathrm{ii}}^{\mathrm{SDM}}\left(\omega_{\mathrm{N}}\right), J_{\mathrm{ii}}^{\mathrm{SDM}}\left(\omega_{\mathrm{h}}\right)$, was measured through three estimators (Table 4). For Interleukin-4, the agreement between predicted NCR and rSDM values is satisfactory for $J_{\mathrm{ii}}^{\mathrm{NCR}}\left(\omega_{\mathrm{N}}\right)$ vs $\int_{\mathrm{ii}}^{\mathrm{SDM}}\left(\omega_{\mathrm{N}}\right)$ and $J_{\mathrm{ii}}^{\mathrm{NCR}}\left(\omega_{\mathrm{h}}\right)$ vs $\int_{\mathrm{ii}}^{\mathrm{SDM}}\left(\omega_{\mathrm{h}}\right)$. In contrast, predicted ${ }_{\mathrm{ii}}^{\mathrm{NCR}}\left(\omega_{\mathrm{h}}\right)$ in Lysozyme correlate rather poorly with $J_{\mathrm{ii}}^{\mathrm{SDM}}\left(\omega_{\mathrm{h}}\right)$ extracted from experimental data (Figure 3 ). This is related to the discrepancies observed between the Overhauser rates $\eta_{\mathrm{NHi}}^{\mathrm{NCR}}$ and $\eta_{\mathrm{NHi}}^{\mathrm{exp}}$ since NOEs reflect the contributions of the spectral density functions at high frequencies. Interestingly, the experimental
Table 4. Comparison between predicted and experimental spectral densities. The spectral densities $J_{\mathrm{ii}}^{\mathrm{SDM}}(0)$ were calculated using $R_{2 \mathrm{i}}^{\text {true }}=R_{2 \mathrm{i}}^{\mathrm{app}}-R_{\text {exi }}$

\begin{tabular}{|lllll|} 
& \multicolumn{2}{c}{ Lysozyme } & \multicolumn{2}{c|}{ Interleukin-4 } \\
& $\rho_{\mathrm{s}}$ & $\rho_{\mathrm{l}}$ & $\rho_{\mathrm{s}}$ & $\rho_{\mathrm{l}}$ \\
\hline$J_{\mathrm{ii}}^{\mathrm{NCR}}(0)$ vs $\int_{\mathrm{ii}}^{\mathrm{SDM}}(0)$ & 0.60 & 0.63 & 0.64 & 0.77 \\
$J_{\mathrm{ii}}^{\mathrm{NCR}}\left(\omega_{\mathrm{N}}\right)$ vs $J_{\mathrm{ii}}^{\mathrm{SDM}}\left(\omega_{\mathrm{N}}\right)$ & 0.63 & 0.67 & 0.60 & 0.80 \\
$J_{\mathrm{ii}}^{\mathrm{NCR}}\left(\omega_{\mathrm{h}}\right)$ vs $J_{\mathrm{ii}}^{\mathrm{SDM}}\left(\omega_{\mathrm{h}}\right)$ & -0.23 & -0.14 & 0.66 & 0.71 \\
\hline
\end{tabular}

$\eta_{\mathrm{NHi}}^{\mathrm{exp}}$ profile is rather flat across the entire protein whilst the predicted $\eta_{\mathrm{NHi}}^{\mathrm{NCR}}$ rates exhibit more pronounced local variations. Neither local nor global root mean square deviations differ significantly in Lysozyme and in Interleukin-4. In the latter, variations of $\eta_{\mathrm{NHi}}^{\mathrm{exp}}$ are reasonably well predicted, as attested by good correlation coefficients.

\subsection{NCR Predictions of Relaxation Rates in the Presence of Anisotropic Overall Diffusion}

As explained above, anisotropic global diffusion affects spin relaxation in a manner that depends on the orientations of the vectors with respect to the overall diffusion tensor. In order to show that the NCR approach is not limited to isotropic overall tumbling, we considered the protein GB3, which is known to exhibit significant anisotropic diffusion. Overall diffusion in GB3 can be described by an axially symmetric diffusion tensor with $D_{\|} / D_{\perp}=1.68 \pm 0.08 .^{[22]}$ Relaxation rates were predicted from Equation (13), by using eight types of vectors in the network, which were extracted from the $\mathrm{X}$-ray structure ${ }^{[23]}$ of protein GB3. The characteristic internal diffusion time $t_{\mathrm{D}}$ was set to $t_{\mathrm{D}}=$ 300 ps.

In order to investigate the implications of diffusional anisotropy, calculations were performed on protein $\mathrm{GB} 3$ using various dynamic models. A comparison of our predictions with experimental measurements ${ }^{[22]}$ is shown in Figure 4. For residue Lys 9 , an exchange contribution $R_{\text {exi }}=1.0 \pm 0.3 \mathrm{~s}^{-1}$ was taken into account. Three situations were investigated: a rigid molecule with anisotropic diffusion, an isotropically tumbling protein with internal dynamics, and a more realistic model of an anisotropically tumbling molecule with internal motions. In the rigid body ( $O$ in Figure 4 ), the predicted rates $R_{2 \mathrm{i}}^{\mathrm{NCR}}$ are uniform in $\beta$-sheets, where $\mathrm{NH}$ vectors are roughly parallel, whereas they show a sawtooth profile in 

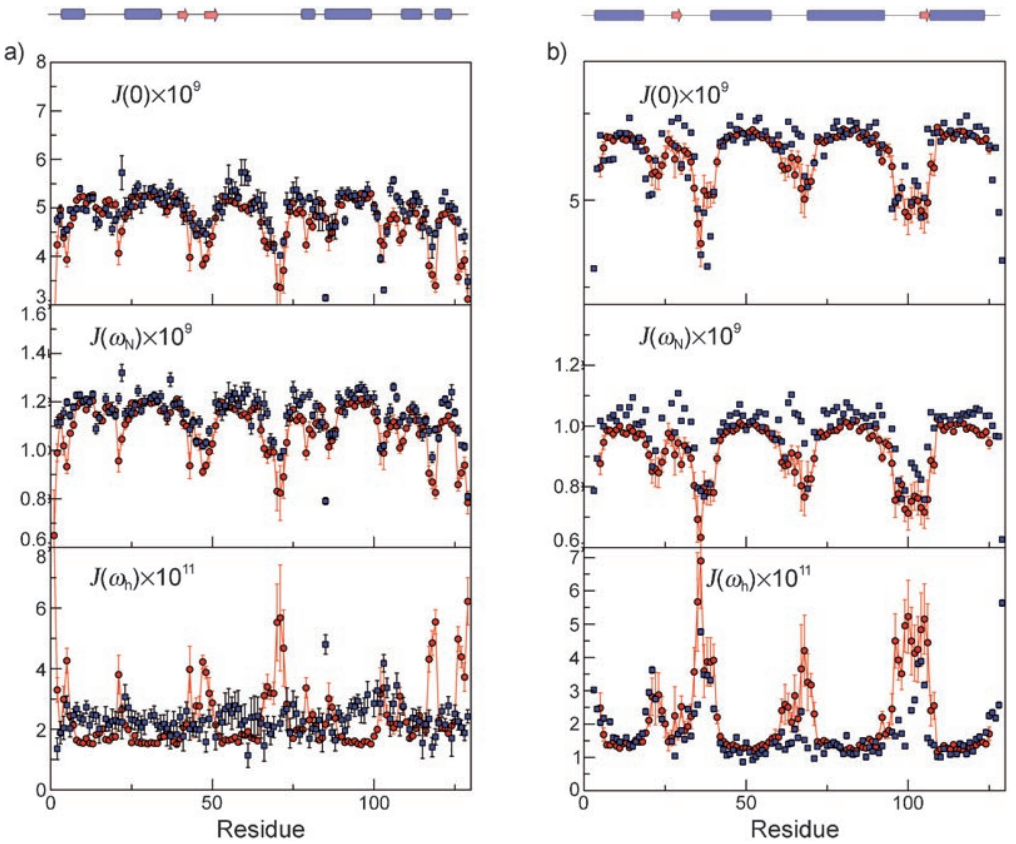

Figure 3. Comparison of the predicted spectral densities $J_{\mathrm{ii}}^{\mathrm{NCR}}(0), J_{\mathrm{ii}}^{\mathrm{NCR}}\left(\omega_{\mathrm{N}}\right), J_{i i}^{\mathrm{NCR}}\left(\omega_{\mathrm{h}}\right)$ (red circles) with the spectral densities obtained by reduced spectral density mapping $J_{\mathrm{ii}}^{\mathrm{SDM}}(0), J_{\mathrm{ii}}^{\mathrm{SDM}}\left(\omega_{\mathrm{N}}\right), J_{\mathrm{ii}}^{\mathrm{SDM}}\left(\omega_{\mathrm{h}}\right)$ (blue squares) in a) Lysozyme and $\mathrm{b}$ ) Interleukin-4. The spectral densities $J_{\mathrm{ii}}^{\mathrm{SDM}}(0)$ were calculated by using $R_{2 \mathrm{i}}^{\text {true }}=R_{2 \mathrm{i}}^{\mathrm{app}}-R_{\mathrm{ex}}$. At the top of the figure secondary structure elements are represented, with $\alpha$-helices as shaded boxes and $\beta$-strands as arrows. (red circles) do the predicted rates $R_{2 \mathrm{i}}^{\mathrm{NCR}}$ reveal a satisfactory agreement with measurements, as attested by a rank-order coefficient $\rho_{s}=0.8$. The predicted values of $R_{1 \mathrm{i}}^{\mathrm{NCR}}, R_{2 \mathrm{i}}^{\mathrm{NCR}}, \eta_{\mathrm{NHi}}^{\mathrm{NCR}}$ are compared with experimental data in Figure $5 \mathrm{a}$. The predicted values of the spectral density functions $J_{\mathrm{ii}}^{\mathrm{NCR}}(0), J_{\mathrm{ii}}^{\mathrm{NCR}}\left(\omega_{\mathrm{N}}\right)$, and $J_{\mathrm{ii}}^{\mathrm{NCR}}\left(\omega_{\mathrm{h}}\right)$ for anisotropic overall tumbling with internal dynamics modelled by NCR are compared to the values obtained from rSDM of the experimental rates in Figure $5 \mathrm{~b}$. Both graphics show very good agreement between predicted and experimental values, which is corroborated by large correlation coefficients $\rho_{\mathrm{s}}$ and $\rho_{\mathrm{l}}$ and rather low root mean square deviation (rmsd) values $\sigma$, shown in Table 5.
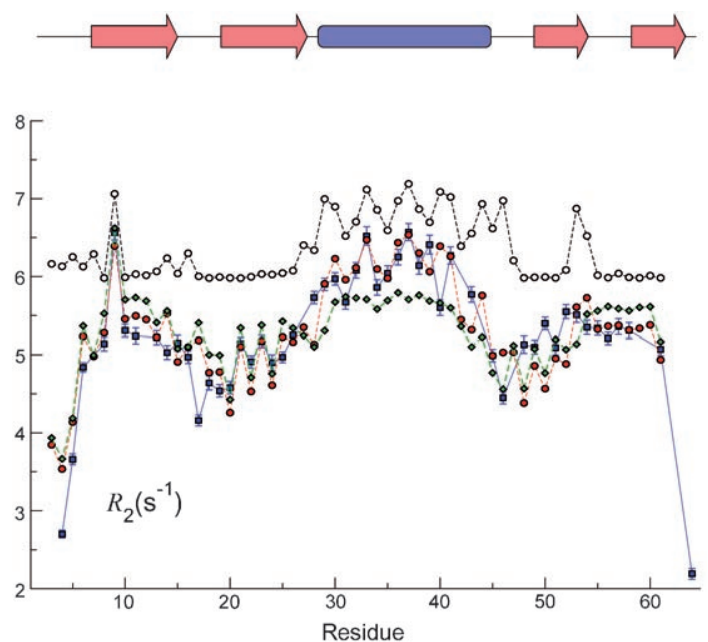

Figure 4. Experimental (blue squares) and predicted transverse relaxation rates $R_{2}$ for protein GB3. The models (NCR + anisotropic tumbling), (rigid body + anisotropic tumbling), and (NCR + isotropic tumbling) are referred to by (red circles), (white circles) and (green diamonds), respectively. At the top of the figure secondary structure elements are represented, with $\alpha$-helices as blue boxes and $\beta$-strands as pink arrows.

$\alpha$-helices. Moreover, relaxation is faster for $\mathrm{NH}$ vectors that are nearly parallel to the principal axis of the diffusion tensor. In the case of overall isotropic diffusion ( $\diamond$ in Figure 4), a non-uniform profile of relaxation rates is predicted, which is typical of the presence of internal dynamics. Clearly, neither of these two naive models is able to reproduce the experimental data. Only when taking global anisotropy and local mobility into account

\section{Discussion}

\subsection{Determination of Characteristic Internal Diffusion Time $t_{\mathrm{D}}$}

The NCR approach allows one to predict ${ }^{15} \mathrm{~N}$ NMR relaxation rates because the dynamic equations determine the time evolution of all the vectors in the network. As explained in Section (2), the timescale of all internal motions in the NCR model is determined by the value $t_{\mathrm{D}}=1 / 6 D$, where the diffusion constant $D$ is common to all rotators. In order to elaborate on this aspect, relaxation rates and spectral density functions were predicted for protein GB3 for different values of $t_{D}$, as shown in Figure 6. It can be seen that $t_{\mathrm{D}}$ only moderately influences the values of $R_{1 \mathrm{i}}^{\mathrm{NCR}}$ and $R_{2 \mathrm{i}}^{\mathrm{NCR}}$. Similarly, the values of $J_{\mathrm{ii}}^{\mathrm{NCR}}(0)$ and $J_{\mathrm{ii}}^{\mathrm{NCR}}\left(\omega_{\mathrm{N}}\right)$ are hardly modified by changes in $t_{\mathrm{D}}$. Alternatively, $\eta_{\mathrm{NHi}}^{\mathrm{NCR}}$ rates are strongly affected by changes in $t_{\mathrm{D}}$, which is related to the fact that these are largely determined by the values of the spectral density function at high frequencies $\left(\omega_{H}, \omega_{H} \pm\right.$ $\omega_{\mathrm{N}}$ ). The variation of $\eta_{\mathrm{NHi}}^{\mathrm{NCR}}$ with $t_{\mathrm{D}}$ is in accordance with the sensitivity of the high-frequency component $J_{\mathrm{ii}}^{N C R}\left(\omega_{\mathrm{h}}\right)$ to $t_{\mathrm{D}}$ in the rSDM analysis of NCR-predicted relaxation rates. The predictions were compared with experiments through the use of the three estimators $\rho_{s}, \rho_{l}$ and $\sigma$, as depicted in Figure 7. These graphs were used to extract a compromise value of $t_{\mathrm{D}}$ ensuring both satisfactory correlation coefficients $\rho_{\mathrm{s}}$ and $\rho_{\mathrm{l}}$ and a low rmsd $\sigma$. The value retained for protein GB3 was $t_{\mathrm{D}}=300 \mathrm{ps}$. Similar investigations showed that the choice of the characteristic internal diffusion time was less critical in Lysozyme and Interleukin-4, and $t_{\mathrm{D}}=300 \mathrm{ps}$ was also retained in these cases. However, this parameter is not as well-defined for Lysozyme and Interleukin-4 as it is for protein GB3. The fact that a wide 

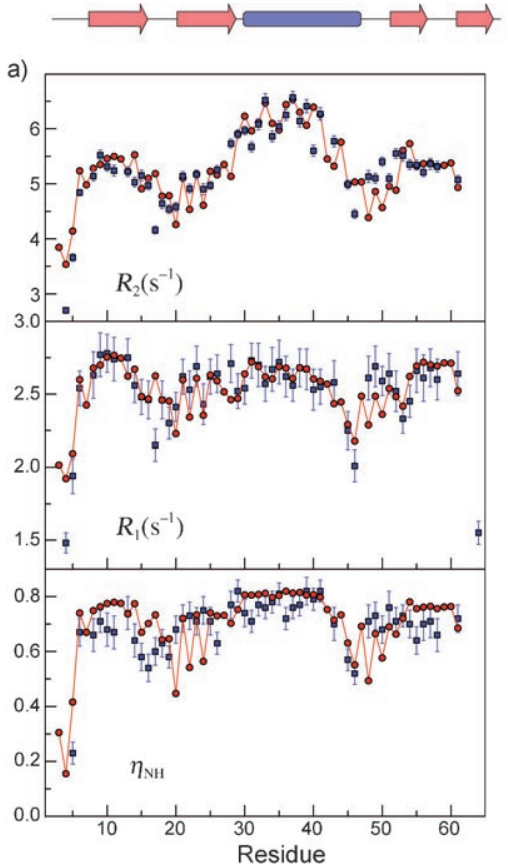

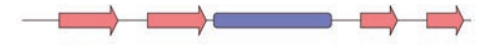

b)

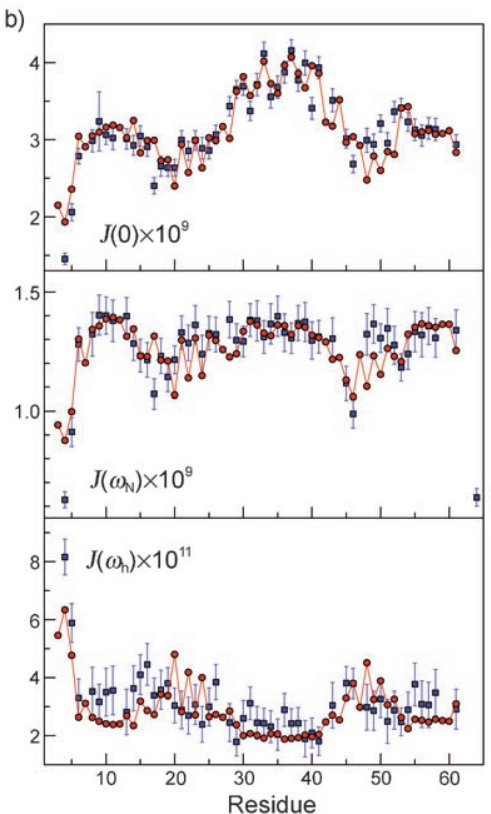

Figure 5. a) Comparison of $R_{1 \mathrm{i}}^{\mathrm{NCR}}, R_{2 \mathrm{i}}^{\mathrm{NCR}}, \eta_{\mathrm{NHi}}^{\mathrm{NCR}}$ (red circles) with $R_{1 \mathrm{i}}^{\exp }, R_{2 \mathrm{i}}^{\text {true }}=R_{2 \mathrm{i}}^{\mathrm{app}}-R_{\mathrm{ex}}, \eta_{\mathrm{NHi}}^{\text {exp }}$ (blue squares) in the anisotropically tumbling protein GB3. b) Comparison of $J_{\mathrm{ii}}^{\mathrm{NCR}}(0), J_{\mathrm{ii}}^{\mathrm{NCR}}\left(\omega_{\mathrm{N}}\right), J_{\mathrm{ii}}^{\mathrm{NCR}}\left(\omega_{\mathrm{h}}\right)$ (red circles) versus $J_{\mathrm{ii}}^{\mathrm{SDM}}(0), J_{i i}^{\mathrm{SDM}}\left(\omega_{N}\right)$, $J_{\mathrm{ii}}^{\mathrm{SDM}}\left(\omega_{\mathrm{h}}\right)$ (blue squares). At the top of the figure secondary structure elements are represented, with $\alpha$-helices as blue boxes and $\beta$-strands as pink arrows.

\begin{tabular}{|c|c|c|c|c|c|c|c|c|c|}
\hline \multirow[t]{2}{*}{ protein GB3 } & \multicolumn{3}{|c|}{$\begin{array}{c}\text { Anisotropic Tumbling } \\
\text { With NCR }\end{array}$} & \multicolumn{3}{|c|}{$\begin{array}{l}\text { Anisotropic Tumbling } \\
\text { No Internal Motions }\end{array}$} & \multicolumn{3}{|c|}{$\begin{array}{c}\text { Isotropic Tumbling } \\
\text { With NCR }\end{array}$} \\
\hline & $\rho_{\mathrm{s}}$ & $\rho_{\mathrm{l}}$ & $\sigma$ & $\rho_{\mathrm{s}}$ & $\rho_{\text {l }}$ & $\sigma$ & $\rho_{\mathrm{s}}$ & $\rho_{\mathrm{l}}$ & $\sigma$ \\
\hline$R_{2 \mathrm{i}}^{\mathrm{NCR}}$ vs $R_{2 \mathrm{i}}^{\text {true }}$ & 0.80 & 0.85 & 0.37 & 0.58 & 0.59 & 1.18 & 0.72 & 0.76 & 0.46 \\
\hline$R_{1 \mathrm{i}}^{\mathrm{NCR}}$ vs $R_{1 \mathrm{i}}^{\exp }$ & 0.68 & 0.79 & 0.14 & 0.13 & 0.04 & 0.50 & 0.59 & 0.73 & 0.17 \\
\hline$\eta_{\mathrm{NHi}}^{\mathrm{NCR}}$ vs $\eta_{\mathrm{NHi}}^{\exp }$ & 0.51 & 0.76 & 0.12 & 0.37 & 0.20 & 0.21 & 0.46 & 0.79 & 0.12 \\
\hline$J_{\mathrm{ii}}^{\mathrm{NCR}}(0)$ vs $J_{\mathrm{ii}}^{\mathrm{SDM}}(0)$ & 0.80 & 0.87 & - & 0.62 & 0.67 & - & 0.68 & 0.72 & - \\
\hline$J_{\mathrm{ii}}^{\mathrm{NCR}}\left(\omega_{\mathrm{N}}\right)$ vs $J_{\mathrm{ii}}^{\mathrm{SDM}}\left(\omega_{\mathrm{N}}\right)$ & 0.67 & 0.78 & - & 0.07 & 0.03 & - & 0.62 & 0.75 & - \\
\hline$J_{\mathrm{ii}}^{\mathrm{NCR}}\left(\omega_{\mathrm{h}}\right)$ vs $J_{\mathrm{ii}}^{\mathrm{SDM}}\left(\omega_{\mathrm{h}}\right)$ & 0.49 & 0.67 & - & 0.41 & 0.36 & - & 0.39 & 0.67 & - \\
\hline
\end{tabular}

range of $t_{\mathrm{D}}$ values is acceptable for these proteins is related to the difficulty of obtaining satisfactory predictions for $\eta_{\mathrm{NHi}}^{\mathrm{NCR}}$.

This strategy, however, is only an empirical way of determining an adequate set of parameters that ensures good agreement with the experiments. In order to gain better insight, we plotted the eigenvalue distribution of the matrix $\mathbf{A}$ of Equation (7). As seen in Equations (7)-(10), the frequency distribution of the motions predicted by the NCR model, that is, the range of the eigenvalues in Equation (10), is scaled by $t_{\mathrm{D}}$. The histogram and the cumulative distribution of the eigenvalues obtained for the three trial values $t_{\mathrm{D}}=100,300,900 \mathrm{ps}$ are represented in Figure 8. A vertical dashed line is drawn at the value $2 \pi \omega_{H}$ to guide the eye. For large values of $t_{\mathrm{D}}$, almost all eigenvalues $\lambda$ are smaller than $2 \pi \omega_{\mathrm{H}}$, with a cumulative distribution close to unity at this frequency. Alternatively, for the short value $t_{\mathrm{D}}=100 \mathrm{ps}$, most eigenvalues are larger than $2 \pi \omega_{H}$.
This seems to indicate that the more numerous the eigenvalues smaller than $2 \pi \omega_{\mathrm{H}}$, the larger their contribution to the spectral density function at frequencies $\omega_{\mathrm{H}}$ and $\omega_{\mathrm{H}} \pm \omega_{\mathrm{N}}$. Thus, $J_{\mathrm{ii}}^{\mathrm{NCR}}\left(\omega_{\mathrm{h}}\right)$ is critically sensitive to this eigenvalue distribution, and is overestimated if $t_{\mathrm{D}}$ is too long, and underestimated if it is too short, as shown in Figure $6 \mathrm{~b}$. An adequate compromise is thus found for an intermediate value $t_{\mathrm{D}}=300 \mathrm{ps}$. An interpretation of these observations is the aim of subsequent work.

\subsection{Local Anisotropy and Relaxation Rates}

Equations (3) and (13) illustrate the fact that the anisotropy of local motions introduced by the NCR potential may contribute to the global correlation functions $C_{\mathrm{ii}}(t)$ only if the overall diffusion is anisotropic. Indeed, inspection of Equation (13) shows that, in addition to the usual term $1-3\left\langle u_{\mathrm{ix}}^{2}+u_{\mathrm{iy}}^{2}\right\rangle \quad$ corresponding to $S_{\mathrm{ii}}^{2}$ in the isotropic case, there is a supplementary term $\varepsilon_{\mathrm{i}}=\left\langle u_{\mathrm{ix}}^{2}-u_{\mathrm{iy}}^{2}\right\rangle$ which also contributes to the asymptotic limit $C_{\mathrm{ii}}^{\mathrm{l}}(\infty)$. Thus, the NCR approach allows one to assess the contribution of local anisotropy to the total spectral density function by calculating $\left\langle u_{\mathrm{ix}}^{2}+u_{\mathrm{iy}}^{2}\right\rangle$ from the terms $\left\langle u_{\mathrm{ix}}^{2}\right\rangle$ and $\left\langle u_{\mathrm{iy}}^{2}\right\rangle$ in the covariance matrix. ${ }^{[1,2]}$ The local anisotropy parameter $\varepsilon_{\mathrm{i}}$ for the protein GB3 is shown in Figure 9. These values should be compared to $\left\langle u_{\mathrm{ix}}^{2}+u_{\mathrm{iy}}^{2}\right\rangle$, which lies between 0.1 and 0.03 for $S_{\mathrm{ii}}^{2}$ in $[0.6,0.9]$. It is seen that the protein exhibits significant anisotropy of local motions along its backbone, particularly in the four $\beta$-strands. By contrast, no significant local anisotropy is observed for the $\mathrm{N}-\mathrm{H}$ vectors in the $\alpha$-helix. However, it is striking that the anisotropy of the local motion does not contribute significantly to the correlation function. Indeed, the correlation functions calculated for protein GB3 by retaining only the terms involving $\left\langle u_{\mathrm{ix}}^{2}+u_{\mathrm{iy}}^{2}\right\rangle$ and $\left\langle u_{\mathrm{ix}}(0) u_{\mathrm{ix}}(t)+u_{\mathrm{iy}}(0) u_{\mathrm{iy}}(t)\right\rangle$ in Equation (13) were indistinguishable from those obtained by including the terms containing $\varepsilon_{\mathrm{i}}$ and yielded exactly the same relaxation rates. Moreover, a model-free analysis performed on rates calculated with and without $\varepsilon_{\mathrm{i}}$ terms yielded the same dynamic parameters (not 
a)

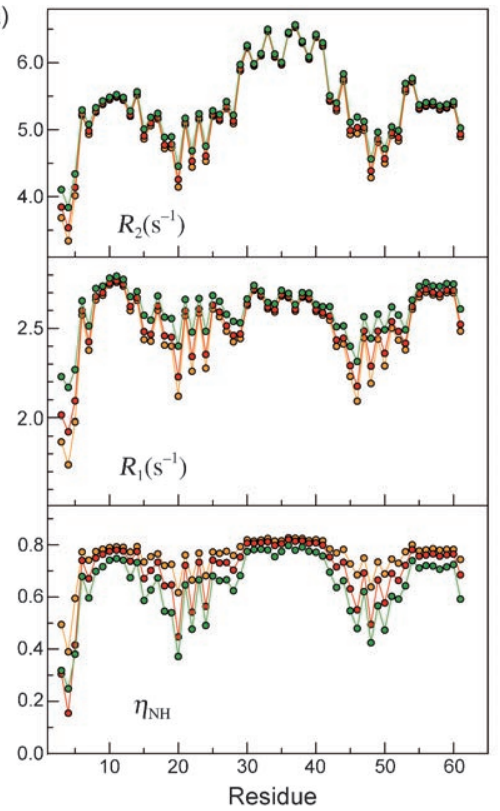

b)

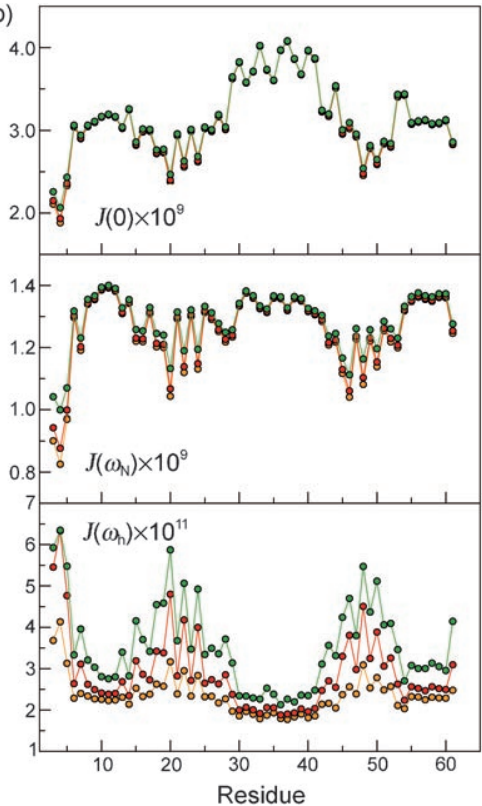

Figure 6. NCR-predicted relaxation rates (left) and spectral densities $J_{i i}^{N C R}(0),{ }_{i i}^{N C R}\left(\omega_{N}\right), J_{i i}^{N C R}\left(\omega_{h}\right)$ (right) of protein GB3 for three different values $t_{\mathrm{D}}=100 \mathrm{ps}$ (yellow); $t_{\mathrm{D}}=300 \mathrm{ps}(\mathrm{red}) ; t_{\mathrm{D}}=900 \mathrm{ps}$ (green).

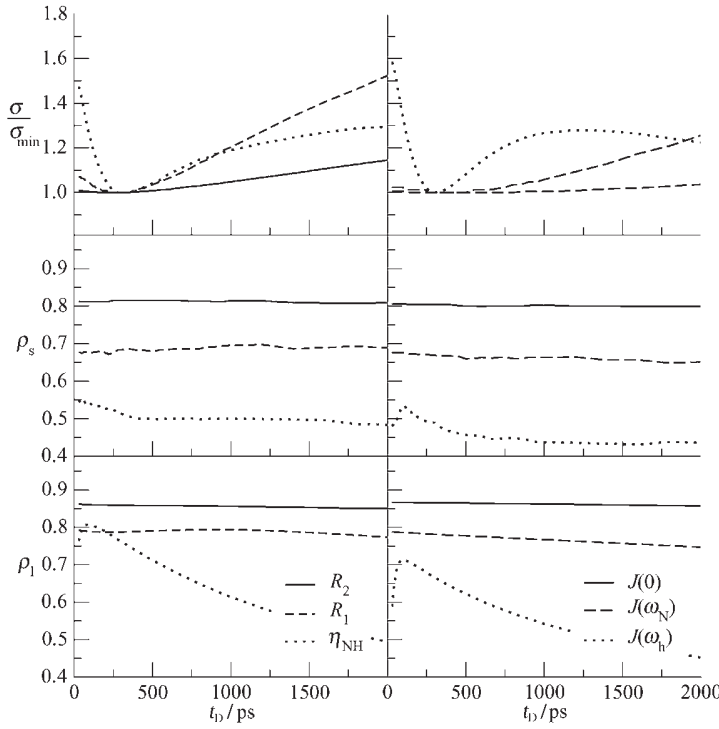

Figure 7. Spearman rank-order $\left(\rho_{\mathrm{s}}\right)$, linear $\left(\rho_{1}\right)$ correlation coefficients, and rmsd $(\sigma)$ obtained from the comparison of $R_{1 \mathrm{Ni}}^{\mathrm{NCR}}, R_{2 \mathrm{Ni}}^{\mathrm{NCR}}$ and $\eta_{\mathrm{NHi}}^{\mathrm{NCR}}$ with experimental values as a function of $t_{\mathrm{D}}$.

shown). This can be understood by analyzing the evolution of the total correlation functions $C_{\text {ii }}(t)$ at times so short that the time dependence of the global diffusion in Equation (13) can be approximated by $e^{-D_{\mathrm{r}} \mathrm{t}} \approx 1-D_{\mathrm{r}} \mathrm{t}$. In this case, one obtains [Eq. (17)]:

$$
\begin{aligned}
& C_{\mathrm{ii}}(t) \approx \sum_{\mathrm{r}=0}^{\mathrm{r}=+2}\left(1-D_{\mathrm{r}} t\right)\left[\xi_{\mathrm{ir}, 1} S_{\mathrm{ii}}^{2}+\xi_{\mathrm{ir}, 2}\left\langle u_{\mathrm{ix}}^{2}-u_{\mathrm{iy}}^{2}\right\rangle^{\text {eq }}\right. \\
& \left.+3 \xi_{\mathrm{ir}, 3}\left\langle u_{\mathrm{ix}}(0) u_{\mathrm{ix}}(t)+u_{\mathrm{iy}}(0) u_{\mathrm{iy}}(t)\right\rangle+\xi_{\mathrm{ir}, 4}\left\langle u_{\mathrm{ix}}(0) u_{\mathrm{ix}}(t)-u_{\mathrm{iy}}(0) u_{\mathrm{iy}}(t)\right\rangle\right]
\end{aligned}
$$

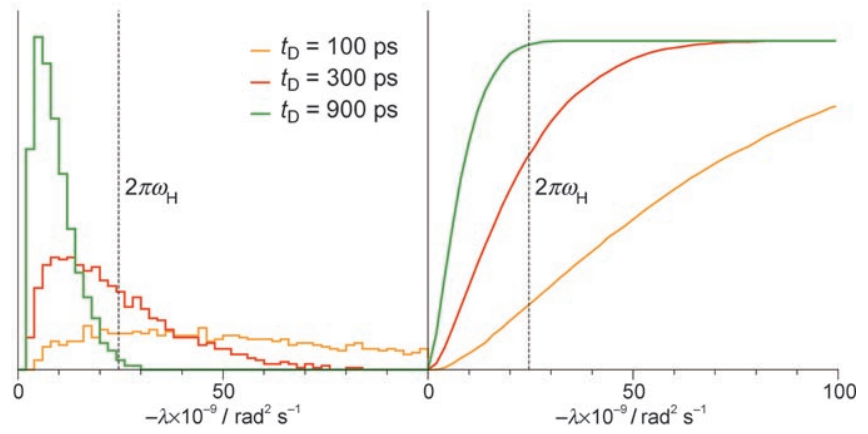

Figure 8. Eigenvalues $\left\{\lambda_{i}\right\}$ of the matrix $\mathbf{A}$ of Equation (7) for $t_{\mathrm{D}}$ values of $100 \mathrm{ps}$ (yellow), $300 \mathrm{ps}$ (red) and $900 \mathrm{ps}$ (green): histogram (left) and cumulative distribution (right).

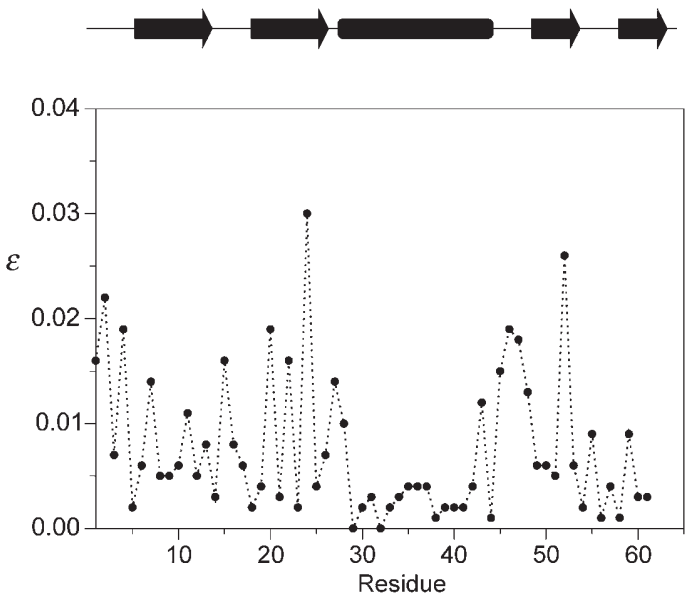

Figure 9. Local anisotropy parameter $\varepsilon_{\mathrm{i}}$ of the local motions calculated for the protein GB3. At the top of the figure secondary structure elements are represented, with $\alpha$-helices as boxes and $\beta$-strands as arrows. 


$$
\begin{aligned}
& \text { Using the relationships } \sum_{\mathrm{r}=0}^{2} \xi_{\mathrm{ir}, 1}=\sum_{\mathrm{r}=0}^{2} \xi_{\mathrm{ir}, 3}=1 \quad \text { and } \\
& \begin{array}{l}
\sum_{\mathrm{r}=0}^{2} \xi_{\mathrm{ir}, 2}=\sum_{\mathrm{r}=0}^{2} \xi_{\mathrm{ir}, 4}=0 \text {, it follows that [Eq, (18)]: } \\
C_{\mathrm{ii}}(t) \approx S_{\mathrm{ii}}^{2}+3\left\langle u_{\mathrm{ix}}(0) u_{\mathrm{ix}}(t)+u_{\mathrm{iy}}(0) u_{\mathrm{iy}}(t)\right\rangle \\
-t \sum_{\mathrm{r}=0}^{\mathrm{r}=+2} D_{\mathrm{r}} \xi_{\mathrm{ir}, 1} S_{\mathrm{ii}}^{2}+3 D_{\mathrm{r}} \xi_{\mathrm{i}, 3}\left\langle u_{\mathrm{ix}}(0) u_{\mathrm{ix}}(t)+u_{\mathrm{iy}}(0) u_{\mathrm{iy}}(t)\right\rangle \\
-t \sum_{\mathrm{r}=0}^{\mathrm{r}=+2} D_{\mathrm{r}} \xi_{\mathrm{ir}, 2}\left\langle u_{\mathrm{ix}}^{2}-u_{\mathrm{iy}}^{2}\right\rangle^{\text {eq }}+D_{\mathrm{r}} \xi_{\mathrm{ir}, 4}\left\langle u_{\mathrm{ix}}(0) u_{\mathrm{ix}}(t)-u_{\mathrm{iy}}(0) u_{\mathrm{iy}}(t)\right\rangle
\end{array}
\end{aligned}
$$

Therefore, significant contributions to the anisotropy of the local motion can be anticipated only when $\sum_{\mathrm{r}=0}^{2} D_{\mathrm{r}} \xi_{\mathrm{ir}, 2}$ or $\sum_{r=0}^{2} D_{r} \xi_{i r, 4}$ are not close to zero, which is expected only in proteins with pronounced overall anisotropic tumbling, $D_{\|} \gg D_{\perp} .^{[9]}$ This allows one to justify a posteriori the model-free analysis of relaxation rates in proteins with moderately anisotropic overall tumbling, where the derivation of an order parameter is based on the assumption of axially symmetric internal motions. ${ }^{[7,24,25]}$ Indeed, we show here that the NCR-predicted contributions of local motional anisotropy to the total correlation function and therefore to the relaxation rates remain negligible in cases where the anisotropy of overall tumbling is moderate, which helps one to understand why an order parameter $S_{\mathrm{ii}}^{2}$ can still be defined.

\section{Conclusions}

Herein, we demonstrate the ability of the NCR approach to predict relaxation rates in several proteins. This approach allows one to predict relaxation rates starting from 3D protein structures. The effect of anisotropic overall tumbling is investigated with application to the case of the protein GB3. Our findings suggest that the measurements of spin relaxation rates due to auto-correlated fluctuations do not provide a truly satisfactory route to investigate the anisotropy of internal motions. However, the use of alternative observables such as residual dipolar couplings or cross-correlated relaxation rates may allow the determination of such contributions.

\section{Acknowledgements}

We would like to thank Anne Dhulesia for fruitful discussions. This work has been supported by the European Union (Research
Training Network "Cross-Correlations" HPRN-CT-2000-00092, the Coordination Action "Focusing NMR on the machinery of life", and the Integrated Infrastructure Initiative "EU-NMR"), the Centre National de la Recherche Scientifique (CNRS), the Université Paris VI, the Ministère de l'Education Nationale, de la Reserche et de la Technologie (MENRT) and the Agence Nationale de la Recherche (ANR).

Keywords: biophysics - coupled rotators - NMR spectroscopy protein folding $\cdot$ protein dynamics

[1] D. Abergel, G. Bodenhausen, J. Chem. Phys. 2005, 123, 204901.

[2] A. Dhulesia, D. Abergel, G. Bodenhausen, J. Am. Chem. Soc. 2007, 129, 4998-5006.

[3] G. Nodet, G. Bodenhausen, D. Abergel, C. R. Chimie, DOI: 10.1016/ j.crci.2007.08.008.

[4] D. Abergel, G. Bodenhausen, J. Chem. Phys. 2004, 121, 761.

[5] A. Abragam, Principles of Nuclear Magnetism, Clarendon, Oxford, 1961.

[6] K. Loth, P. Pelupessy, G. Bodenhausen, J. Am. Chem. Soc. 2005, 127, 6062.

[7] J. M. Schurr, H. P. Babcock, B. S. Fujimoto, J. Magn. Reson. Ser. B 1994, $105,211-224$

[8] D. Korzhnev, M. Billeter, A. Arseniev, V. Orekhov, Prog. Nucl. Magn. Reson. Spectrosc. 2001, 38, 197-266.

[9] V. A. Daragan, K. H. Mayo, J. Phys. Chem. B 1999, 103, 6829.

[10] P. Dosset, D. Marion, M. Blackledge, Copyright 1999, Institut de Biologie Structurale J. P. EBEL CEA-CNRS, Laboratoire de Resonance Magnetique nucleaire, Grenoble, 2000.

[11] Scilab, a free scientific software package.

[12] J. W. Peng, G. Wagner, J. Magn. Reson. 1992, 98, 308-332.

[13] J. W. Peng, G. Wagner, Biochemistry 1992, 31, 8571-8586.

[14] A. Farrow, O. Zhang, J. Forman-Kay, L. E. Kay, Biochemistry 1995, 34, 868-878.

[15] J.-F. Lefèvre, K. T. Dayie, J. W. Peng, G. Wagner, Biochemistry 1996, 35, 2674-2686.

[16] A. Farrow, O. Zhang, A. Szabo, D. A. Torchia, L. E. Kay, J. Biomol. NMR 1995, 6, 153-162.

[17] M. Buck, J. Boyd, C. Redfield, D. MacKenzie, D. Jeenes, D. Archer, C. Dobson, Biochemistry 1995, 34, 4041-4055.

[18] C. Redfield, J. Boyd, L. J. Smith, R. Smith, C. Dobson, Biochemistry 1992, 31, 10431-10437.

[19] H. Schwalbe, S. B. Grimshaw, A. Spencer, M. Buck, J. Boyd, C. M. Dobson, C. Redfield, L. J. Smith, Protein Sci. 2001, 10, 677-688.

[20] T. Müller, T. Dieckmann, W. Sebald, H. Oschkinat, J. Mol. Biol. 1994, 237, 423-436.

[21] W. H. Press, B. P. Flannery, S. Teukolsky, W. T. Vetterling. Numerical Recipes, Cambridge University Press, Cambridge, 1989.

[22] M. L. Tillet, M. Blackledge, J. P. Derrick, L.-Y. Lian, T. J. Norwood, Protein Sci. 2000, 9, 1210-1216.

[23] W. D. Derrick, J. Mol. Biol. 1994, 243, 906-918.

[24] G. Lipari, A. Szabo, J. Am. Chem. Soc. 1982, 104, 4546.

[25] G. Lipari, A. Szabo, J. Am. Chem. Soc. 1982, 104, 4559.

Received: October 31, 2007 